
\title{
28 Research Suare \\ Enhancing early breast milk for sick and preterm neonates admitted at regional referral hospitals in Dar es Salaam, Tanzania
}

\section{Regina Hyera}

Muhimbili University of Health and Allied Sciences

\section{Evelyne Assenga}

Muhimbili University of Health and Allied Sciences

Theodore Kazimoto

Muhimbili University of Health and Allied Sciences

Germana Leyna

Muhimbili University of Health and Allied Sciences

Francis Mchomvu

Comprehensive Community Based Rehabilitation Tanzania

Antke Zuechner ( $\nabla$ antke.zuechner@web.de )

Comprehensive Community Based Rehabilitation in Tanzania https://orcid.org/0000-0001-5569-276X

\section{Research}

Keywords: Very low birth weight, hypoxic ischemic encephalopathy, early breastmilk feeding, necrotizing enterocolitis, feeding guidelines, Tanzania

Posted Date: June 7th, 2020

DOl: https://doi.org/10.21203/rs.3.rs-32417/v1

License: (c) (1) This work is licensed under a Creative Commons Attribution 4.0 International License. Read Full License 


\section{Abstract}

Background: Feeding is a cornerstone in the management of sick or preterm neonates and early initiation of breastfeeding is the single most effective intervention associated with reduced neonatal mortality. However, enteral feeding is often delayed due to haemodynamic instability and the perceived risk of necrotizing enterocolitis (NEC). Alternative routes for early breast milk administration are either oropharyngeal colostrum or minimal enteral nutrition. One strategy to overcome delays in enteral feeds is the implementation of standardized feeding guidelines.

Methods: This study was conducted in three regional hospitals in Dar es Salaam, consisting of a historical control group at baseline and an intervention group after implementation of locally developed feeding guidelines. Neonates were consecutively recruited and followed to a maximum of 28 days of life. The controls received routine standard care, while the intervention group received early enteral feeding. The outcomes measured were NEC, time to regain birth weight and neonatal mortality. Odds ratio was used to determine the association between feeding and outcomes, statistical significance was considered when $p$-value $\leq 0.05$.

Results: 292 neonates were enrolled in this study, 163 (55.8\%) controls at baseline and 129 (44.2\%) after the intervention. Study participants comprised of 130 (44.5\%) neonates with very low birth weight and 162 (55.5\%) with hypoxic ischemic encephalopathy. The mean age of initiating feeding was $45.34 \pm$ 21.58 SD hours amongst the controls and $8.43 \pm 3.02$ SD hours in the intervention group. Overall, $1.4 \%$ (4 cases) of the neonates were diagnosed with NEC, $1.8 \%$ (3 cases) in the control group and $0.8 \%$ (1 case) in the intervention group ( $P=0.4 ; 95 \% \mathrm{Cl}$ : 0.25-23.3). The mean duration of regaining birth weight was significantly reduced from $11.26 \pm 4.34$ SD days among controls to $8.25 \pm 2.96$ SD days in the intervention group ( $p=0.000 ; 95 \% \mathrm{Cl}: 1.61-4.41)$. Mortality in this high-risk groups of neonates remained high (40.8\%), without significant difference between the control (41.7\%) and intervention group (39.5\%).

Conclusions: Neonates who received early breast milk feeding following the implementation of feeding guidelines significantly regained birth weight earlier. No association was demonstrated between early breast milk feeding and increased NEC or mortality.

\section{Background}

Despite remarkable success in the reduction of child mortality over the last decades, neonatal mortality rates have remained high, especially in low income countries. Most of these deaths are preventable by known, evidence based interventions which need to be implemented at scale [1-3]. Feeding is a cornerstone in the management of sick or preterm neonates admitted to a neonatal care unit [4] and early initiation of breastfeeding is one of the most cost-effective interventions associated with reduced neonatal mortality [5-7]. During the neonatal period the nutritional demand is high due to rapid growth and brain development. Adequate nutrition improves growth and neurodevelopmental outcome [8]. Early 
enteral feeding provides a neonate with colostrum, mother's first milk, which is rich in immune-protective and growth factors. It also promotes gut maturation, intestinal enzymes and hormonal responses $[9,10]$. Furthermore, previous studies have demonstrated that delayed initiation of enteral feeds leads to significant postnatal growth failure and adverse neonatal outcomes, especially in low resource settings where total parenteral nutrition for neonates is not readily available [11-13].

However, there is a wide variety of enteral feeding practices for sick and preterm infants since universal feeding guidelines are non-existent $[14,15]$. Enteral feeding is often delayed in settings without intensive care facilities due to haemodynamic instability and the perceived risk of feeding intolerance or necrotizing enterocolitis (NEC). This is contrary to findings from several studies which have shown that early introduction of mother's milk is not associated with an increased risk of NEC $[16,17]$. For critically ill neonates or very low birth weight (VLBW) infants, there are alternative routes of administrating mother's milk, either by oropharyngeal colostrum (OPC) or minimal enteral nutrition (MEN) [9, 17-22]. Therefore,

the implementation of standardized and locally adapted feeding guidelines is one strategy to overcome the delay in enteral feeds and has demonstrated improved nutritional outcomes $[8,23]$.

The Maternal and Newborn Healthcare Capacity Building Team (MHCB) of the local non-governmental organization Comprehensive Community Based Rehabilitation in Tanzania (CCBRT) in Dar es Salaam is supporting several public hospitals and health centers in the region by providing regular training and mentoring to the medical staff. In order to address neonatal mortality MHCB aims to introduce low cost and low technology strategies to improve neonatal care. One of the apparent problems in the neonatal units of the three regional hospitals was the delayed initiation of breastmilk feeding for critical ill neonates and VLBW infants. In discussions with the local teams of neonatal health care providers and colleagues from Muhimbili University for Health and Allied Sciences (MUHAS), it was agreed to jointly work out a project to enhance early breast milk feeding for sick and preterm neonates. It was envisioned that through the training and use of standardized feeding guidelines, we could gather scientific evidence of the effect of early feeding of sick and preterm neonates and to dispel the reservations to early neonatal feeding amongst health care personnel in the regional hospitals.

\section{Methods}

\section{Study design and setting}

This was a hospital-based, non-blinded, quasi-experimental study using a historical control group and an intervention group which was conducted at Amana, Mwananyamala and Temeke Hospitals, which are the three regional referral hospitals of Dar es Salaam, Tanzania. Each of these hospitals has a neonatal care unit admitting between 2800 and 4400 neonates every year.

\section{Project implementation}

A baseline survey of the historical control group was carried out from October 2018 to December 2018 using a structured questionnaire to collect demographic data of enrolled neonates and their mothers and 
to determine the time until critically ill neonates receive their first administration of mother's milk, regain their birth weight and duration of hospital stay. At the same time adverse outcomes were monitored and documented.

While baseline data were being collected, a two-day workshop with the paediatricians responsible for the neonatal wards was conducted to develop locally adjusted standardized feeding guidelines for very sick and preterm neonates (see annex 1). A consensus was reached after review of the current literature and available feeding protocols from other centres. In a second workshop the local neonatal nursing staff created standard operating procedures (SOPs) on breastfeeding counselling, expressing breast milk, inserting a nasogastric tube (NGT) and feeding by cup or NGT.

After implementation of the new feeding guidelines and SOPs another period of 3 months data collection was conducted from February 2019 to April 2019 using the same data tool to obtain the corresponding data amongst the intervention group.

\section{Study subjects}

The study recruited neonates with VLBW and moderate to severe HIE admitted between October 2018 and April 2019. Neonates who were admitted $>24$ hours of life, born to HIV positive mothers who opted not to breastfeed, with features suggesting gastrointestinal malformations and those whose mothers declined consent, were excluded from the study. In a study done by Abdelmaaboud et al [24] in Quatar from 2009 to 2011 the proportion of NEC among neonates with delayed feeding was $12.9 \%$. Using the power of $80 \%$, at $95 \%$ confidence level with the assumption that introduction of early feeding will reduce the proportion of NEC by $80 \%$ (from $12.9-2.6 \%$ ), the estimated sample size was 292. All eligible neonates were enrolled consecutively on admission until the sample size was reached.

\section{Data collection methods and instruments}

A pre-tested structured questionnaire was used to collect participant's information including sociodemographic characteristics, feeding pattern, adverse events and neonatal outcomes. Dependent variables assessed were NEC as a primary outcome, neonatal mortality, time to regain birth weight and duration of hospital stay as secondary outcomes; while time to initiation of feeding was the independent variable.

Study participants were enrolled during admission according to the inclusion criteria of birth weight 1000-1500 g for VLBW and Thompson HIE score > 10 for neonates with moderate to severe birth asphyxia. Body weight, gestational age (determined by Ballard score), HIE score and vital signs were recorded (see annex 2). Neonates in the historical control group started feeding after stabilization based on the decision of the attending paediatrician.

Study participants enrolled in the intervention group started early breast milk administration within 24 hours either by the means of OPC or MEN according to the newly implemented feeding guidelines as decided by the attending paediatrician. OPC was administered by giving $0.2 \mathrm{ml}$ in each cheek using a 
$2 \mathrm{ml}$ syringe. MEN was started with $10 \mathrm{ml} / \mathrm{kg}$ /day through NGT within the first 24hours and advanced at the rate of $20 \mathrm{ml} / \mathrm{kg} /$ day from the second day of life until reaching full enteral feeding of $150 \mathrm{ml} / \mathrm{kg} / \mathrm{day}$. Body weight was recorded every morning by trained research assistants.

If a neonate presented with abdominal distension, bilious vomiting and bloody diarrhea a plain abdominal $x$-ray was done to establish the occurrence of NEC which was diagnosed both clinically and radiologically using Bell's criteria whereby stage $2 \mathrm{~B}$ was regarded as significant. A neonate with confirmed NEC was managed by the attending paediatrician following standard management guidelines.

During the intervention, participants' safety was assessed through the monitoring for adverse events by a Data Safety and Monitoring Board (DSMB) comprised of independent reviewers. The DSMB consisted of one pediatrician, an epidemiologist and one biostatistics expert and met two weeks after data collection of the intervention group had started.

\section{Data management and statistical analysis}

Data was entered, cleaned and analyzed using SPSS version 20. It was an intention to treat analysis. The categorical variables NEC and mortality were presented using proportions. Continuous variables which were length of hospital stay and duration of regaining birth weight were presented using mean (standard deviation). Chi-square test or when appropriate Fisher's exact test was used to compare statistical significance in outcomes. Odds ratio (OR) was used to determine association between independent variable (feeding type) and dependent variables. The results were considered of statistical significance when $p$-value was $\leq 0.05$.

\section{Results}

\section{Baseline characteristics of study participants}

A total of 292 neonates were enrolled in this study, 163 (55.8\%) in the control group and 129 (44.2\%) in the intervention group. Study participants comprised of $130(44.5 \%)$ neonates with VLBW and 162 (55.5\%) with moderate to severe HIE. Males accounted for $53.4 \%$. The two groups were comparable in their baseline characteristics as shown in Table 1. 
Table 1

Socio-demographic characteristics of the study participants

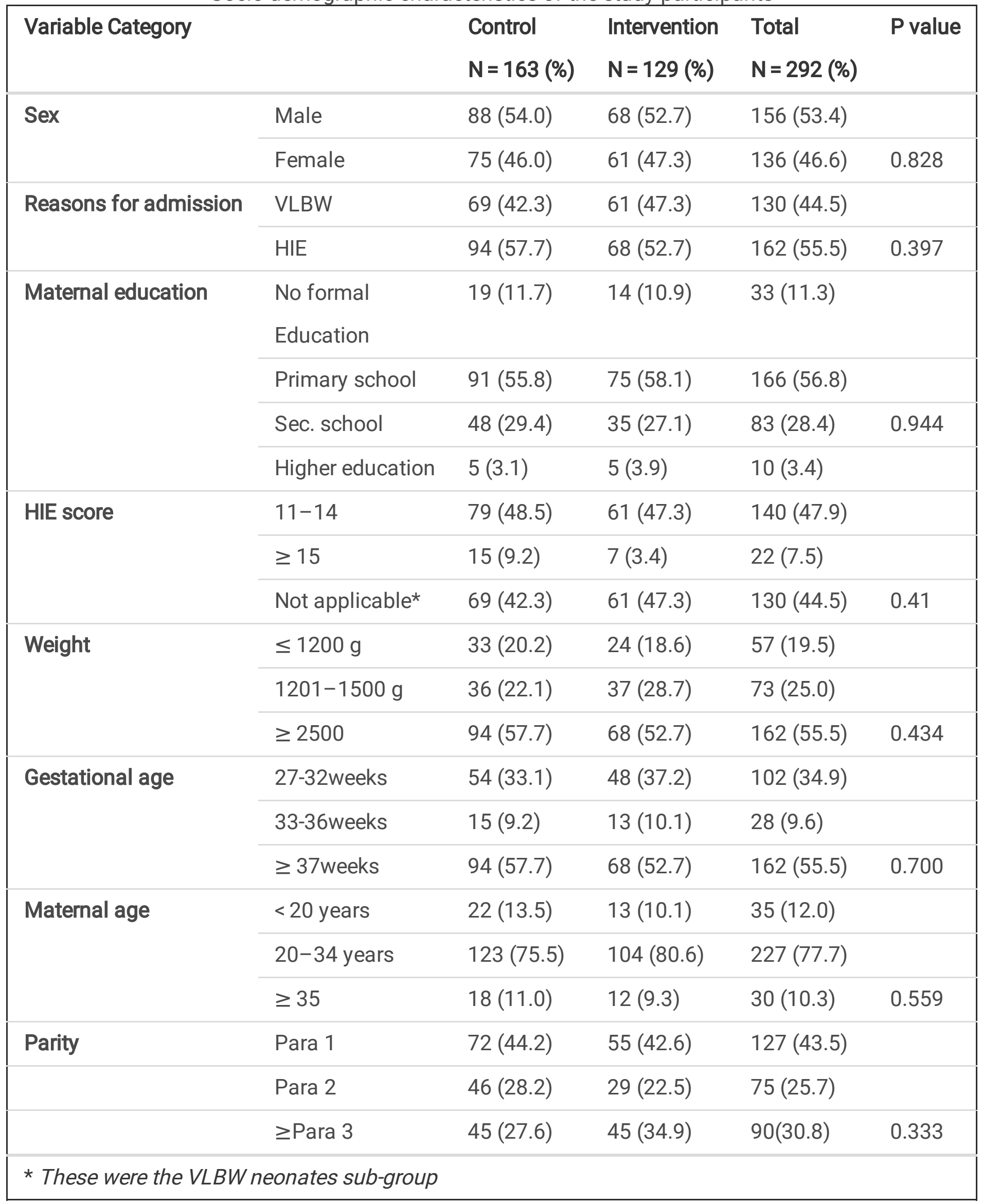


Feeding characteristics of study participants

The mean age of initiating feeding was $45.34 \pm 21$.58SD SD hours in the control group compared to 8.43 $\pm 3.02 \mathrm{SD}$ hours amongst the intervention group after the implementation of feeding guidelines as depicted in Table 2.

Table 2

Feeding characteristics of study participants

\begin{tabular}{|c|c|c|c|c|c|}
\hline \multirow[t]{2}{*}{ Variable Category } & & Control & Intervention & Total & \multirow{2}{*}{$\begin{array}{l}\mathrm{P} \\
\text { value }\end{array}$} \\
\hline & & $N=163(\%)$ & $N=129(\%)$ & $\begin{array}{l}\mathrm{N}=292 \\
(\%)\end{array}$ & \\
\hline \multirow{2}{*}{$\begin{array}{l}\text { Started feeding before } \\
\text { end point }\end{array}$} & Yes & 114 (69.9) & $124(96.1)$ & $\begin{array}{l}237 \\
(81.5)\end{array}$ & \\
\hline & No & $49(30.1)$ & $5(4.7)$ & $54(18.5)$ & 0.00 \\
\hline \multirow[t]{3}{*}{$\begin{array}{l}\text { Mean age started feeding } \\
\text { (hours) }\end{array}$} & $\begin{array}{l}\text { All } \\
\text { neonates }\end{array}$ & $\begin{array}{l}45.34 \pm 21.58 \\
S D\end{array}$ & $\begin{array}{l}8.43 \pm 3.02 \\
S D\end{array}$ & & 0.00 \\
\hline & \multirow{2}{*}{$\begin{array}{l}\text { VLBW } \\
\text { HIE }\end{array}$} & $\begin{array}{l}38.12 \pm 14.08 \\
\text { SD }\end{array}$ & $\begin{array}{l}8.25 \pm 3.14 \\
\text { SD }\end{array}$ & & 0.00 \\
\hline & & $\begin{array}{l}51.58 \pm 25.24 \\
\text { SD }\end{array}$ & $\begin{array}{l}8.59 \pm 2.49 \\
\text { SD }\end{array}$ & & 0.00 \\
\hline
\end{tabular}

\section{The mean age of regaining birth weight amongst the study participants}

The mean time for regaining birth weight was significantly reduced from $11.26 \pm 4.34$ SD days among the control group to $8.25 \pm 2.96$ SD days in the intervention group $(p=0.000)$ as shown in Table 3 .

Table 3

Mean age of regaining birth weight amongst the study participants

\begin{tabular}{|lllll|}
\hline Mean age regained birth weight (days) & Control & Intervention & Pvalue & $\mathbf{9 5 \%} \mathbf{C l}$ \\
& $\mathbf{n = 5 4}$ & $\mathbf{n = 5 6}$ & & \\
\hline Overall & $11.26 \pm 4.34 S D$ & $8.25 \pm 2.96 S D$ & $\mathbf{0 . 0 0 0}$ & $1.61-4.41$ \\
\hline VLBW & $12.18 \pm 4.64 S D$ & $8.81 \pm 3.23 S D$ & $\mathbf{0 . 0 0 0}$ & $1.54-5.20$ \\
\hline HIE & $8.64 \pm 1.50 S D$ & $7.16 \pm 1.98 S D$ & 0.025 & $0.2-2.77$ \\
\hline
\end{tabular}

Duration of hospital stay

The mean duration of hospital stay was $9.02 \pm 7.83$ SD days in the control group and $8.85 \pm 7.12$ SD days among the intervention group, but the difference was not statistically significant $(p=0.85)$. The mean duration of hospital stay was slightly longer among neonates with VLBW, $12.84 \pm 9.48$ SD days in control 
group vs $11.95 \pm 8.04$ SD days in the intervention group $(p=0.57)$ than among neonates with HIE who had a mean stay of $6.4 \pm 4.3$ SD days in the control group vs $5.94 \pm 4.3$ SD days in the intervention group $(p=0.49)$.

\section{Proportion of mortality among study participants}

The overall mortality was $40.8 \%$ (119 patients) with $41.7 \%$ (68 patients) among controls and $39.5 \%$ (51 patients) in the intervention group, but the difference was not statistically significant $(p=0.72, O R=0.91$; $95 \% \mathrm{Cl}$ 0.57-1.46). There was an apparently higher mortality among VLBW in the intervention group $(37.7 \%)$, when compared to the control group $(33.3 \%)$, but again no statistical significance $(p=0.6)$. Controls with HIE showed a mortality of $47.9 \%$, while in the intervention group $41.2 \%(p=0.4)$

\section{Factors associated with neonatal mortality}

In the subgroup of VLBW infants, the mortality was significantly increased in neonates with a lower birth weight of $\leq 1200 \mathrm{~g}$ compared to those with $>1200-1500$ grams $(50.9 \%$ vs. $23.3 \% ; p=0.001)$. In the subgroup of neonates with $\mathrm{HIE}$, those scoring $\geq 15$ denoting severe $\mathrm{HIE}$, had a significantly higher mortality rate compared to those with a score of $<15(90.9 \%$ vs. $37.9 \% ; p=0.000)$.

\section{Discussion}

The aim of this project was to enhance early breast milk administration for sick and preterm neonates admitted to the neonatal wards of the regional hospitals in Dar es Salaam by developing and implementing standardized feeding guidelines adapted to the local context and the same time controlling for potentially harmful effects. Therefore, the project was embedded in a study which determined the effect of early feeding amongst the intervention group on the incidence of NEC and mortality amongst neonates with VLBW and HIE when compared to a historical control group before implementation of the feeding guidelines.

The mean age for initiation of feeding was $45.34 \pm 21.58$ SD hours in the control group compared to 8.43 \pm 3.02 SD hours in the intervention group showing that early breast milk administration was successfully established following the implementation of newly developed feeding guidelines. This corresponds well to previous studies which described improved enteral feeding initiation through implementation of standardized feeding protocols $[8,23]$.

Our findings revealed that the overall proportion of NEC was only $1.4 \%$ (4 cases) among the study participants without significant difference between the control and intervention groups, but the study was underpowered to detect statistical significance. This low incidence of NEC is similar to studies from Iran [25] and Jordan [26]. However, studies examining the age at onset of NEC reported a mean age of 6-7 days for the occurrence of NEC in more mature preterm infants and a possible delay in the onset of NEC to more than 14 days for smaller infants with lower gestational age $[27,28]$. Considering the high mortality in this study it was not possible to assess all enrolled study participants for the occurrence of 
NEC as the outright features of NEC may have not yet developed, especially in the highest risk group of VLBW infants with a birth weight of less than 1200 gram who had a significant increased mortality.

Early initiation of breast milk feeding significantly reduced the duration of regaining birth weight more than three days in the intervention group when compared to the controls. This finding demonstrates the benefit of early enteral feeding in the prevention of postnatal growth failure and is consistent with studies from Uganda [12], Iran [25] and India [11, 29]. However, this did not correspond with a shortened hospital stay as shown in previous studies $[11,25]$ and may be explained by other factors such as neonatal sepsis which may impact on the early stabilization of the neonates.

The relatively high mortality rate among the controls (41.5\%) and intervention group (39.5\%) didn't show a significant association to the initiation of early feeding. A birth weight of $\leq 1200 \mathrm{~g}$ and Thompson HIE score of $\geq 15$ were independent predictors of mortality among neonates with very low birth weight and perinatal asphyxia respectively. The high mortality rate for high risk neonates in our study corresponds well to results from other low income countries, where neonatal intensive care facilities are not yet available $[26,30,31]$.

Limitations of this study: A quasi-experimental design with historical controls can lead to selection bias due to change in the study population over time. Neonates who were referred to a tertiary hospital were not followed to determine their outcomes, and this might result in an underestimation of the proportion of necrotizing enterocolitis and mortality.

\section{Conclusions}

The implementation of newly developed guidelines through involvement of local paediatricians successfully enabled the enhancement of early breast milk administration for sick and preterm neonates. Neonates who received early feeding significantly regained birth weight earlier than those with delayed feeding. However, early feeding did not shorten the duration of hospital stay. Early breast milk feeding for high-risk neonates also did not show an association with increased NEC or mortality, but our study was underpowered to measure these safety outcomes.

\section{Recommendations}

Healthcare workers should be trained on how to initiate and support early breast milk feeding for sick and preterm neonates to ensure neonates quickly regain their birth weight. A randomized study with a large sample size and high power is recommended to further confirm the safety of early provision of mother's milk via OPC/MEN among high risk neonates in a low resource setting.

\section{Abbreviations}

CCBRT 
Comprehensive Community Based Rehabilitation in Tanzania; DSMB:Data Safety and Monitoring Board; HIE:hypoxic ischaemic encephalopathy; MEN:minimal enteral nutrition; MHCB:Maternal and Neonatal Healthcare Capacity Building; MUHAS:Muhimbili University for Health and Allied Sciences; NEC:necrotizing enterocolitis; NGT:nasogastric tube; OPC:oropharyngeal colostrum; OR:Odds ratio; SOP:standard operating procedure; VLBW:very low birth weight;

\section{Declarations}

\section{Ethics approval and consent to participate}

Ethical clearance was obtained from the MUHAS Institutional Review Board and permission to conduct this study was sought from Temeke, Amana and Mwananyamala municipal authorities and the respective hospitals. The study was registered in the Pan African Clinical Trial Registry (www.pactr.org) under the identification number PACTR201810856025709.

\section{Consent for publication}

Not applicable.

\section{Availability of data and materials:}

The datasets used and/or analysed during the current study are available from the corresponding author on reasonable request.

\section{Competing interests}

The authors declare that they have no competing interests.

\section{Funding}

This project was funded by Laerdal Foundation, Stavanger, Norway under their funding line "Saving lives at birth".

\section{Authors' contributions}

$\mathrm{RH}$ provided major contributions in concept, data collection, literature review and drafting the manuscript. EA contributed to concept, data analysis, literature review and drafting the manuscript. TK and GL contributed to concept and data analysis. FM contributed to concept and data collection. AZ contributed to concept, literature review and manuscript. All authors read and approved the final manuscript. 


\section{Acknowledgements}

We are very grateful for the trustful collaboration between Muhimbili University of Health and Allied Sciences, CCBRT and the regional hospitals of Dar es Salaam. The neonatal teams of all institutions made this project possible with their valuable contributions.

\section{Declarations}

Written informed consent was obtained from mothers of eligible neonates. Participants had identification number during data collection which could not be linked back to the participants' hospital details. All participants continued to receive the standard of care offered in the respective hospitals.

\section{References}

1. Levels and trends in Child Mortality 2019. https://www.unicef.org/media/60561/file/UN-IGME-childmortality-report-2019.pdf

2. Althabe F, Bergel E, Cafferata ML, et al. Strategies for improving the quality of health care in maternal and child health in low- and middle-income countries: an overview of systematic reviews. Paediatric and Perinatal Epidemiology 2008; 22: 42 - 60

3. Rollins N, Doherty T. Improving breastfeeding practices at scale. The Lancet 2019: e292

4. Dutta S, Singh B, Chessell L, et al. Guidelines for Feeding Very Low Birth Weight Infants. Nutrients $2015 ; 7,423-442$

5. Smith ER, Hurt L, Chowdhury R, et al. Delayed breastfeeding initiation and infant survival: A systematic review and meta-analysis. PLOS ONE 2017; 12 (7): e0180722

6. Strømmen K, Blakstad W, Moltu J, et al. Enhanced Nutrient Supply to Very Low Birth Weight Infants is Associated with Improved White Matter Maturation and Head Growth. Neonatology 2015; 107 (1): 68-75

7. Su BH. Optimizing nutrition in preterm infants. Pediatrics and Neonatology $2014 ; 55$ (1): 5-13

8. McCallie KR, Lee HC, Mayer O, et al. Improved outcomes with a standardized feeding protocol for very low birth weight infants. Journal of Perinatology 2011; 31: S61 - S67

9. Örs R. The practical aspects of enteral nutrition in preterm infants. J Pediatr Neonat Individual Med 2013; 2 (1): 35-40

10. Sondheimer JM. A critical perspective on trophic feeding. J Pediatr Gastroenterol Nutr 2004; 38 (3): 237- 238

11. Nangia S, Bishnoi A, Goel A, et al. Early Total Enteral Feeding in Stable Very Low Birth Weight Infants: A Before and After Study. Journal of Tropical Pediatrics 2018; 64: 24 - 30 
12. Nakubulwa C, Muslime V, Namiiro FB, et al. Delayed initiation of enteral feeds is associated with postnatal growth failure among preterm infants managed at a rural hospital in Uganda. BMC Pediatrics 2020; 20: 86

13. Konnikova Y, Zaman MM, Makda M, et al. Late Enteral Feedings Are Associated with Intestinal Inflammation and Adverse Neonatal Outcomes. PLOS ONE 2015; 10 (7); e0132924

14. Berti E, Puglia M, Perugi S, et al. Feeding Practices in Very Preterm and Very Low Birth Weight Infants in an Area where a Network of Human Milk Banks is in Place. Frontiers in Pediatrics 2018; 6: 387

15. Moreno Algarra MC, Fernandez Romero V, Sanchez Tamayo T, et al. Variability in enteral feeding practices of preterm infants among hospitals in the SEN1500 Spanish neonatal network. An Pediatr 2017; 87 (5): $242-252$

16. Morgan J, Young L, Mcguire W. Delayed introduction of progressive enteral feeds to prevent necrotising enterocolitis in very low birth weight infants. Cochrane Database Syst Rev. 2014; (12): CD001970

17. Meinzen-Derr J, Poindexter B, Wrage L, et al. Role of human milk in extremely low birth weight infants' risk of necrotizing enterocolitis or death. J Perinatol 2009; 29 (1): 57-62

18. Sondheimer JM. A critical perspective on trophic feeding. J Pediatr Gastroenterol Nutr 2004; 38 (3): 237-238.

19. Agarwal R, Aggarwal R, Deorari AK, Paul VK. Minimal enteral nutrition. Indian J Pediatr . 2001; 68 (12): 1159-60.

20. Morgan J, Young L, Mcguire W. Delayed introduction of progressive enteral feeds to prevent necrotising enterocolitis in very low birth weight infants. Cochrane Database Syst Rev. 2014; 2014 (12):CD001970

21. Rodriguez NA, Meier PP, Groer MW, Zeller JM. Oropharyngeal administration of colostrum to extremely low birth weight infants: Theoretical perspectives. J Perinatol 2009; 29 (1): 1-7

22. Lee J, Kim HS, Jung YH, et al. Oropharyngeal colostrum administration in extremely premature infants: An RCT. World Rev Nutr Diet 2016; 114: 56-7.

23. Patole S, De Klerk N. Impact of standardised feeding regimens on incidence of neonatal necrotising enterocolitis: a systematic review and meta-analysis of observational studies. Arch Dis Chikd Fetal Neonatal Ed. 2005; 90 (2): F147 - F 151

24. Abdelmaaboud $M$, Mohammed A. A randomized controlled trial on early versus late minimal enteral feeding in preterm growth-restricted neonates with abnormal antenatal Doppler studies. J Neonatal Perinatal Med. 2012;5 (2):155-62

25. Sallakh-Niknezhad A, Bashar-Hashemi F, Satarzadeh N, et al. Early versus Late Trophic Feeding in Very Low Birth Weight Preterm Infants. Iran J Pediatr 2012; 22 (2): 171-176

26. Al-Lawama M, Abdelghani T, Badran E. Mortality and Short-Term Outcomes of Very Low Birth Weight Infants at a Tertiary Care Center in Jordan: Comparison with Other Countries. Pediatrics \& Therapeutics 2017, 7 (1): 1000311 
27. Yee WH, Soraisham AS, Shah VS, et al. Incidence and Timing of Presentation of Necrotizing Enterocolitis in Preterm Infants. Pediatrics 2012; 129 (2): 298 - 304

28. González-Rivera R, Culverhouse RC, Aaron Hamvas A, et al. The Age of Necrotizing Enterocolitis Onset: An Application of Sartwell's Incubation Period Model. J Perinatol. 2011; 31 (8): 519-523

29. Tewari V, Dubey SK, Kumar R, Vardhan S, Sreedhar CM, Gupta G. Early versus Late Enteral Feeding in Preterm Intrauterine Growth Restricted Neonates with Antenatal Doppler Abnormalities: An OpenLabel Randomized Trial. J Trop Pediatr. 2018; 64:4-14.

30. Okello F, Egiru E, Acom L, et al. Reducing preterm mortality in eastern Uganda: the impact of introducing low-cost bubble CPAP on neonates < 1500 g. BMC Pediatrics 2019; 19: 31

31. Belizan JM, McClure EM, Goudar SS, et al. Neonatal deaths in Low- to Middle Income Countries: A Global Network Study. American Journal of Perinatology 2012; 29 (08): 649-656

\section{Supplementary Files}

This is a list of supplementary files associated with this preprint. Click to download.

- supplement4.jpg

- supplement5.jpg

- supplement6.pdf 